\title{
Interferência mútua entre atividade visual e atividade motora em jovens e idosos
}

\section{Mutual interference between a visual and a motor task in young and elderly subjects}

\author{
Patrícia Pereira dos Santos Teixeiral, Mariana Callil Voos², Mariane Silva Andrade Machadol, \\ Lílian Zanchetta Castelli', Luiz Eduardo Ribeiro do Valle, Maria Elisa Pimentel Piemonte ${ }^{4}$
}

Estudo desenvolvido no ICB/ USP - Instituto de Ciências Biomédicas da Universidade de São Paulo, São Paulo, SP, Brasil

1 Fisioterapeutas

2 Doutoranda em Neurociências e Comportamento no Instituto de Psicologia da USP

3 Prof. Dr. do ICB/USP

4 Profa. Dra. do Depto. de Fisioterapia, Fonoaudiologia e Terapia Ocupacional da Faculdade de Medicina da USP

ENDEREÇO PARA

CORRESPONDÊNCIA

Mariana C. Voos

Fofito/ FMUSP

Rua Cipotânea 51 Cidade

Universitária

05360-160 São Paulo SP

e-mail: marivoos@usp.br

APRESENTAÇÃO

out. 2007

ACEITO PARA PUBLICAÇÃO

abr. 2008
Resumo: Como o envelhecimento provoca dificuldade na capacidade de dividir a atenção, este estudo visou verificar, em jovens e idosos: (1) a eventual interferência entre uma tarefa visual e uma motora; (2) se essa interferência (caso exista) ocorre de forma diferente no desempenho de jovens e idosos; (3) se as tarefas propostas têm correlação com testes validados, de seqüência alfanumérica (TMT) e de levantar e caminhar cronometrado (TLCC). A tarefa visual consistiu na verbalização do reconhecimento de duas figuras iguais ou diferentes apresentadas rapidamente. A tarefa motora consistiu na alternância de passos do chão a uma plataforma fixa de $10 \mathrm{~cm}$ de altura. As tarefas foram avaliadas isoladas (tarefa-simples) e associadas (tarefa-dupla) em dois grupos: 10 jovens ( $23 \pm 2,8$ anos) e 10 idosos ( $68,8 \pm 8,6$ anos). Na tarefa visual, os jovens fizeram menos erros que os idosos $(p<0,001)$; nos dois grupos não houve maior número de erros na tarefa-simples quando comparada à tarefa-dupla. Na tarefa motora, os idosos tiveram pior desempenho na tarefa-dupla em relação à tarefasimples $(p=0,009)$. Houve correlação positiva entre o TMT e o número de alternâncias de passos $(p<0,05)$ e correlação negativa entre o número de alternâncias de passos e o TLCC $(p<0,05)$. A tarefa motora não interferiu na tarefa visual em jovens nem em idosos, mas a tarefa visual diminuiu a velocidade da tarefa motora dos idosos. A proposição das tarefas sem atribuir primariedade a uma ou outra permitiu detectar essas interferências.

Descritores: Análise e desempenho de tarefas; Atividade motora; Envelhecimento; Percepção visual

ABSTRACT: Since aging brings about difficulty in dividing attention, this study aimed at verifying, in youth and aged: (1) the possible interference between a visual and a motor task; (2) whether such interference varies between young and elderly subjects; (3) whether there is correlation between the proposed tasks and the trail making test (TMT) and the timed up-and-go test (TUGT). The visual task measured the ability to state whether two quickly presented figures were same or different. The motor task consisted on alternating steps from the ground to a $10 \mathrm{~cm}$-high platform. Tasks were assessed both as single-task (isolated) and dual-task (simultaneous) in two groups: 10 young people (aged $23 \pm 2.8$ ) and 10 elderly (aged 68.8 \pm 8.6 ). In the visual task, young volunteers presented less errors than the elderly $(p<0.001)$; in both groups no increase in the number of errors was detected at dual-task when compared to the singletask. At the motor task the elderly presented lower speed in dual-task as compared to the single-task $(p=0.009)$. TMT correlated positively to the number of alternations of step $(p<0.05)$ and TUGT correlated negatively to the number of alternations of step $(p<0.05)$. For both young and elderly, the motor task did not interfere on the visual task; however, the elderly showed lesser speed in the motor task when associated to the visual task. The non-assignment of primality to either task proved useful to detect these interferences.

Key words: Aging; Motor activity; Task performance and analysis; Visual perception 


\section{INTRODUÇÃO}

A associação entre tarefas visuais e motoras é comum e necessária nas atividades diárias, como dirigir, atravessar a rua, tomar conta de crianças, usar o computador, passear com o cachorro... Muitos trabalhos discutem o desempenho visual-motor em experimentos com tarefa-dupla ${ }^{1-4}$. Uma tarefa primária é apresentada ao voluntário e testada na condição isolada e, posteriormente, associada a uma tarefa secundária. A tarefa secundária pode evidenciar mudanças na tarefa primária.

Quando duas tarefas são associadas, há maior probabilidade de uma ou ambas serem realizadas com menor velocidade e maior número de erros, o que é chamado de interferência mútua negativa ${ }^{3}$. Quanto mais complexa for a tarefa secundária, maior será a interferência negativa, ou seja, a piora na tarefa primária ${ }^{2,5}$. Por outro lado, se a tarefa primária for de alta complexidade, a adição de uma tarefa secundária simples poderá acarretar perda de desempenho.

Quando há interferência negativa entre tarefas, assume-se que ambas competem para a mesma classe de recursos de processamento de informação no sistema nervoso, pois processar recursos é algo limitado e compartiIhado. A demanda de atenção reflete o grau de competição de resposta entre representações corticais sobrepostas que participam das duas tarefas associadas $^{6}$. Se duas tarefas são mediadas pela mesma área cortical, há piora de desempenho quando são realizadas ao mesmo tempo (hipótese do campo cortical). Se uma população de neurônios está engajada em uma atividade específica, não estará disponível para desempenhar uma atividade concorrente ${ }^{7}$. A interferência irá ocorrer quando a capacidade de recursos centrais disponíveis for excedida $^{4}$.

Muitos artigos mostram que idosos apresentam dificuldade de dividir a atenção entre duas tarefas ${ }^{5,8-15}$. Isso ocorre devido a défices sensoriais e perceptuais $^{16}$, aumento do tempo de reação da resposta frente ao estímu- lo ${ }^{10,17}$, ou redução da habilidade de planejamento e seleção de estratégias motoras ${ }^{8}$, principalmente quando é solicitada a associação a tarefas cognitivas ${ }^{5,13,18}$; ou ainda a perda de memória de longo prazo e memória operacional ${ }^{19}$.

Alterações de memória operacional, atenção e flexibilidade mental são bem detectadas pelo teste de seqüência alfanumérica, também conhecido como teste das trilhas (TMT, sigla do inglês trail making test) ${ }^{20,21}$, que mede o tempo necessário para o sujeito ligar em seqüência 25 círculos contendo números ou letras e números. Foi encontrada correlação entre desempenho no TMT, mobilidade funcional e risco de quedas em idosos ${ }^{21}$, o que também se relaciona à velocidade de realização do teste de levantar e caminhar cronometrado (TLCC, em inglês TUGT) ${ }^{22}$. O TLCC é uma medida de mobilidade funcional que quantifica em segundos o tempo que o indivíduo leva para levantar de uma cadeira (com assento a cerca de 46 $\mathrm{cm}$ do solo), caminha três metros, vira, volta e se senta novamente. O paciente é instruído a realizar o trajeto o mais rápido possível. Indivíduos com tempo de realização acima de 13 segundos apresentam maior risco de que$\mathrm{da}^{23}$. O TMT ${ }^{20}$ e o TLCC ${ }^{22}$ são amplamente utilizados em idosos e apresentam grande confiabilidade e validade inter e intra-observadores ${ }^{20-22}$.

Um estudo ${ }^{17}$ analisou idosos atravessando a rua e concluiu que a dificuldade de associar o ato motor de atravessar a rua ao de olhar os carros que se aproximam ocorre devido à redução da capacidade de dividir a atenção. Idosos são dependentes da visão quando andam e olham mais para o chão durante a marcha. Pedestres idosos têm maior dificuldade de atravessar a rua em segurança ${ }^{17}$.

A marcha apresenta alto custo de atenção, porém, como é uma prática comum no dia-a-dia, não inibe a capacidade de realizar outras atividades ao mesmo tempo, tendo caráter automático ${ }^{14}$. Com o envelhecimento, o controle rítmico de passos deixa de ser realizado predominantemente por regiões subcorticais e espinhais e passa a ser gerenciado por regiões corticais, também envolvidas em tarefas cognitivas. Logo, a interferência causada pela competição de demandas de atenção é mais evidente em idosos ${ }^{11}$. Além de alterações na marcha, idosos têm dificuldades em outras tarefas cotidianas que requerem divisão de atenção, como dirigir ${ }^{16}$ e deambular em ambientes externos ${ }^{9,12,24}$.

Estudos sobre associação de tarefas costumam determinar arbitrariamente uma tarefa como primária e outra como secundária; nesses casos, a generalização dos achados para atividades diárias poderia não ser adequada, já que não parece possível estipular peso maior, por exemplo, para uma tarefa motora em relação a uma tarefa visual; para atravessar a rua, a alternância de passos e o equilíbrio dinâmico são tão importantes quanto a detecção de obstáculos e veículos em nosso trajeto. Para melhor compreensão dos mecanismos envolvidos em tarefas-duplas, seria necessário o estudo da interação entre tarefas motora e visual sem atribuir primazia a uma ou outra. E, para avaliar o desempenho de idosos, é útil compará-lo ao de jovens. Além disso, para avaliar o desempenho em tarefa-dupla é conveniente verificar se este apresenta correlação com o de testes funcionais validados na literatura, que medem a capacidade de realizar tarefas visualmotoras. Frente ao exposto, os objetivos deste estudo foram verificar:

(1) a eventual interferência entre uma tarefa visual de detecção, discriminação e comparação de dois estímulos e uma tarefa motora de alternância de passos do chão a uma plataforma;

(2) se essa interferência (caso exista) ocorre de forma diferente no desempenho de jovens e idosos;

(3) se o desempenho nos testes realizados apresenta correlação com o desempenho em outros dois testes, o TMT e o TLCC.

\section{METODOLOGIA}

Participaram deste estudo dez voluntários idosos saudáveis (cinco homens), com 60 a 85 anos $(68,8 \pm 8,6$ 
anos de idade), pareados com dez voluntários jovens (cinco homens) com 20 a 35 anos (23 $\pm 2,8$ anos de idade). Foram adotados como critérios de inclusão: escolaridade de 8 a 16 anos, pontuação no miniexame do estado mental (MEEM) ${ }^{26}$ maior que 25 e visão normal ou corrigida com lentes (acuidade visual de longe medida pelo teste de Snellen, visão de perto medida pelo teste de leitura e presença de escotoma avaliada pela grade de Amsler ${ }^{27}$. Os critérios de exclusão foram: uso habitual de jogos eletrônicos, participação prévia em outros experimentos semelhantes a este e problemas musculoesqueléticos, cardiovasculares, respiratórios ou neurológicos.

Os participantes foram esclarecidos quanto aos objetivos do estudo e os que consentiram em participar assinaram um termo de consentimento livre e esclarecido. O estudo foi aprovado pela Comissão de Ética do Instituto de Ciências Biomédicas da Universidade de São Paulo (ICB/USP). O estudo foi conduzido em uma sala com isolamento acústico e iluminação reduzida, no laboratório de Psicofisiologia do ICB/USP. Foram feitas investigações clínicas nos voluntários sobre os critérios de inclusão e exclusão.

Materiais

Foram utilizados: um computador com o aplicativo MEL2 ${ }^{\circledR}$; uma plataforma de $10 \mathrm{~cm}$ de altura, fixa no chão; e anteparos para ajuste da altura do monitor conforme a altura dos voluntários. Foram aplicados dois instrumentos, o miniexame do estado mental $(\mathrm{MEEM})^{26}$ e o teste de seqüência alfanumérica (TMT); este é dividido em duas partes: na parte A, o voluntário deve conectar em ordem crescente, traçando retas, círculos contendo 25 números espalhados randomicamente $(1,2,3 \ldots)$; na parte $B$, há maior demanda cognitiva, pois o voluntário deve seguir uma seqüência crescente de números e letras $(1, A, 2, B, 3, C \ldots)$. $A$ pontuação é dada pela subtração do tempo gasto na parte B menos tempo gasto na parte $\mathrm{A}$.

\section{Procedimentos}

A tarefa visual foi realizada com base na parte 2 do teste UFOV (Useful field of view test) ${ }^{16}$. Consistia na nomeação de dois estímulos simultâneos, um apresentado no centro da tela do computador e outro na periferia (a dez graus do centro, em uma das oito possíveis posições demarcadas em sentido horário), ambos com duração de 150 ms. A atividade era constituída por 32 tentativas, com duração total média de 2,5 minutos. A tela do computador, de fundo cor cinza, trazia no centro uma cruz de fixação e oito retângulos brancos circundandoa (luminância de $20 \mathrm{~cd} / \mathrm{m}^{2}, 2,2^{\circ} \mathrm{de}$ altura por $3,6^{\circ}$ de largura e $0,04^{\circ}$ de borda). Após $1750 \mathrm{~ms}$ aparecia um estímulo de duração de 150 ms no centro da tela (sobre a cruz) e, simultaneamente, outro dentro de um dos retângulos periféricos. Os estímulos podiam ser um caminhão $\left(1,5^{\circ}\right.$ de largura, $0,5^{\circ}$ de altura anterior, $0,9^{\circ}$ de altura posterior) ou um ônibus $\left(1,5^{\circ} \mathrm{de}\right.$ largura, $0,5^{\circ}$ de altura), ambos com luminância de $20 / \mathrm{m}^{2}$. As possibilidades de aparecimento dos estímulos eram: ônibus no centro e na periferia; ônibus no centro e caminhão na periferia; caminhão no centro e na periferia; e caminhão no centro e ônibus na periferia. O estímulo da periferia podia aparecer em qualquer um dos oito retângulos (Figura 1).
Os voluntários mantinham-se em bipedestação a $70 \mathrm{~cm}$ da tela do computador. A altura da tela foi ajustada para que os olhos ficassem no nível da cruz, no centro da tela. Foram orientados a manter os olhos na cruz durante todo o experimento, deixando sua atenção difusa para conseguir reconhecer os estímulos apresentados no centro e na periferia da tela. Os participantes deviam falar em voz alta se os estímulos eram iguais ou diferentes. Após a exibição dos dois alvos, o programa era interrompido até que o/ a participante respondesse ("iguais" ou "diferentes"). Foi proporcionada retroalimentação auditiva (beep) a cada resposta correta. Após cada tentativa, um examinador, que não tinha acesso à tela do teste, registrava a resposta com o mouse e uma nova tentativa era iniciada. Caso não houvesse resposta em 10 segundos, o programa emitia um novo estímulo e a resposta era considerada errada. O programa fornece o número de erros de cada participante.

A tarefa motora consistia na alternância de passos do chão a uma plataforma com superfície antiderrapante, de $10 \mathrm{~cm}$ de altura, fixa ao solo, localizada à frente dos voluntários. Foi solicitado que realizassem a tarefa o mais rápido possível e olhassem para frente durante a atividade. O tempo da atividade motora era igual ao tem-

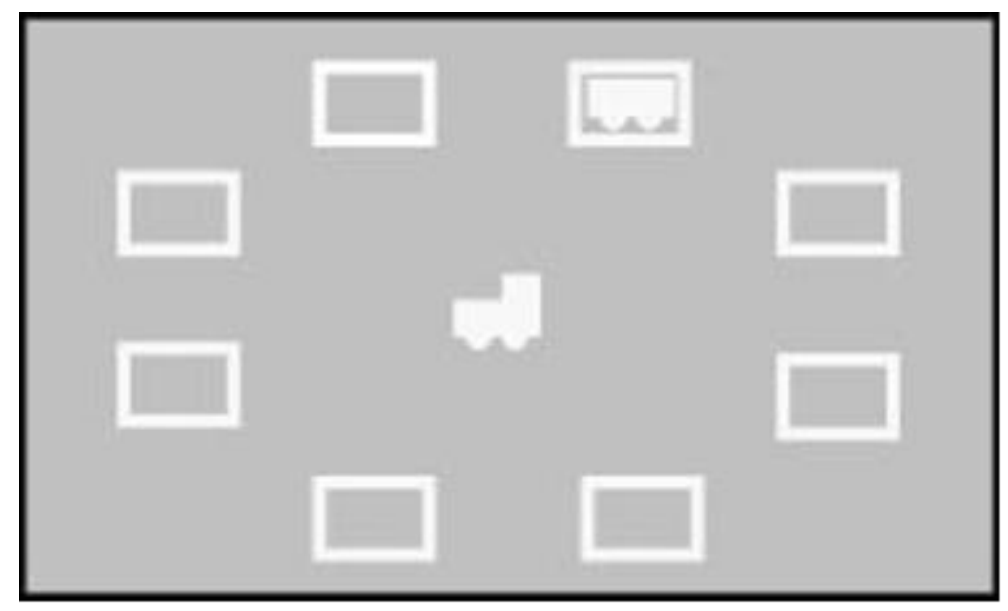

Figura 1 Tarefa visual: imagem apresentada na tela do computador; um ônibus ou um caminhão apareciam no centro e em um dos oito retângulos (estímulos simultâneos, de 150 ms) (Parte 2 do UFOV adaptada de Ball \& Owsley ${ }^{16}$ ) 
po que os voluntários haviam levado para realizar a atividade visual. As alternâncias dos passos sobre a plataforma foram filmadas e posteriormente contadas e registradas pelo examinador.

Todos os voluntários receberam detalhada explicação sobre o experimento. Foram aplicados então, nesta ordem, $1^{\circ}$ o teste TLCC, $2^{\circ}$ treino da tarefa visual (TVtr), $3^{\circ}$ treino da tarefa motora (TMtr), 4ㅇ TMT-parte A, 5 tarefa visual (TV), 6 o tarefa motora (TM), 7은 TMT-parte B, 8을 tarefas visual e motora associadas (TD, tarefa-dupla), 9 $M E E M, 10$ 을 repetição da tarefa motora (TMrep).

Foram alternadas tarefas mais aeróbicas (motora e tarefa-dupla) com tarefas menos cansativas (tarefa visual, TMT partes A e B, MEEM), para maior conforto dos voluntários. Por isso, apesar de a pontuação no MEEM ter sido usada como critério de inclusão, o teste foi o nono da seqüência. Se tivesse havido algum voluntário com pontuação abaixo de 25 , teria realizado todo o experimento normalmente, mas seus dados seriam descartados da análise estatística. A tarefa motora foi repetida ao final do experimento para avaliar se o cansaço interferiu no desempenho; em caso afirmativo, seria observada diminuição do número de alternâncias de passo por segundo.

A pressão arterial dos voluntários foi mensurada em repouso e após as tarefas motoras e a tarefa dupla. Caso a pressão arterial de algum dos voluntários tivesse chegado a 150 x 100 $\mathrm{mmHg}$, o experimento teria sido interrompido por segurança, mas isso não aconteceu.

\section{Análise estatística}

Para testar a possibilidade de ocorrência de interferência negativa mútua entre as tarefas, o número de passos por segundo na ausência e na presença da tarefa visual e o número de erros na tarefa visual na ausência e na presença da tarefa motora foram submetidos a análises de variância (ANOVAs). As mesmas análises foram utilizadas para comparar o desempe- nho de jovens e idosos. Também foram feitos testes de correlação de Spearman para verificar se o desempenho na tarefa motora apresentou correlação com o TLCC e se o desempenho na tarefa visual apresentou correlação com o TMT. O nível de significância adotado foi de $5 \%$ $(\alpha=0,05)$.

\section{RESULTADOS}

A análise dos resultados na tarefa visual evidenciou que os jovens apresentaram menor número de erros $(6,6 \pm 3,5)$ que os idosos $(14,8 \pm 4,1)$ $[\mathrm{F} 1,18=56,16 ; p<0,001]$. Houve diferença entre os testes $[\mathrm{F} 2,36=3,89$; $p=0,030]$ : a comparação post hoc mostrou que o resultado no treino da tarefa visual isolada (TVtr) foi pior $(p=0,034)$ que na tarefa visual associada à motora (TD); mas não houve menor número de erros na tarefa-simples quando comparada à tarefa-dupla, portanto não ocorreu interferência negativa da tarefa motora sobre a visual. Não foi encontrada relação entre grupos e testes $[F 2,36=0,42$; $p=0,660]$ : os grupos de jovens e de idosos apresentaram comportamento semelhante no treino (TVtr), na tare- fa-simples (TV) e na tarefa-dupla ( $\mathrm{Fi}$ gura 2)

Quanto à tarefa motora, os idosos apresentaram uma média de 1,06 $\pm 0,4$ passos por segundo e os jovens, 1,47 $\pm 0,3$ passos por segundo $[\mathrm{F} 1,18=10,31$; $p=0,005]$. Houve diferença entre os testes $[\mathrm{F}=3,54=9,01 ; p<0,001]$ : a comparação post hoc mostrou que o desempenho no treino da tarefa motora (TMtr) foi pior do que na própria tarefa TM $(p<0,001)$, evidenciando aprendizado (Figura 3). O desempenho na tarefa-dupla (motora associada à visual) também foi pior do que na TMsimples $(p=0,002)$. Houve interação entre grupos e testes $[\mathrm{F} 3,54=4,47$; $p=0,007]$. A comparação post hoc mostrou que, entre os jovens, o desempenho no treino da tarefa-simples (TMtr) foi pior que na última (teste de fadiga) $(p<0,001)$. Nos idosos, o desempenho foi melhor na tarefa-simples (TM) quando comparada à tarefa-dupla $(p=0,047)$, e pior na tarefa-dupla quando comparada à última realização da tarefa motora (TMrep, teste de fadiga) ( $p=0,009)$ (Figura 3). Enquanto os jovens melhoraram o desempenho na tarefa motora na condição tarefa-dupla, os idosos apresentaram menor número de alternâncias de pas-

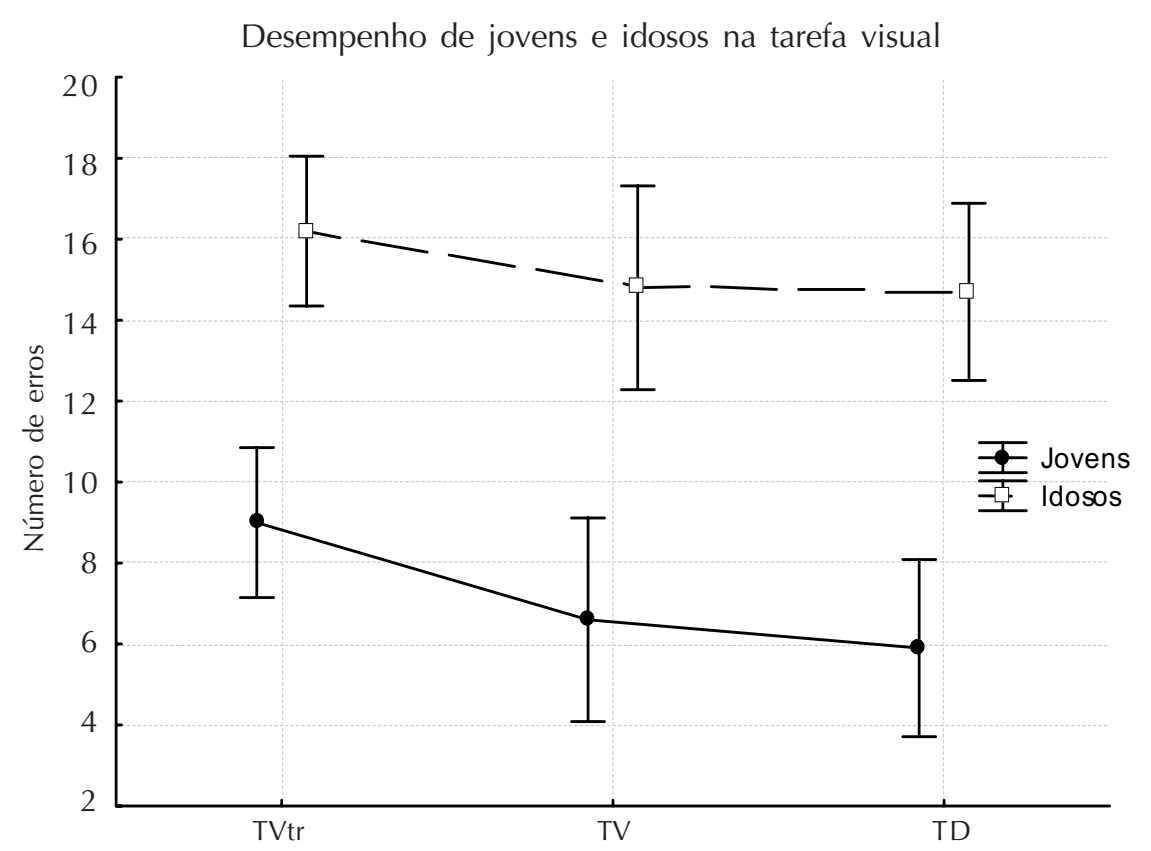

Figura 2 Desempenho médio de jovens e idosos na tarefa visual. TVtr = treino da tarefa visual; TV = tarefa visual; TD = tarefa visual associada à tarefa motora (tarefa-dupla) 


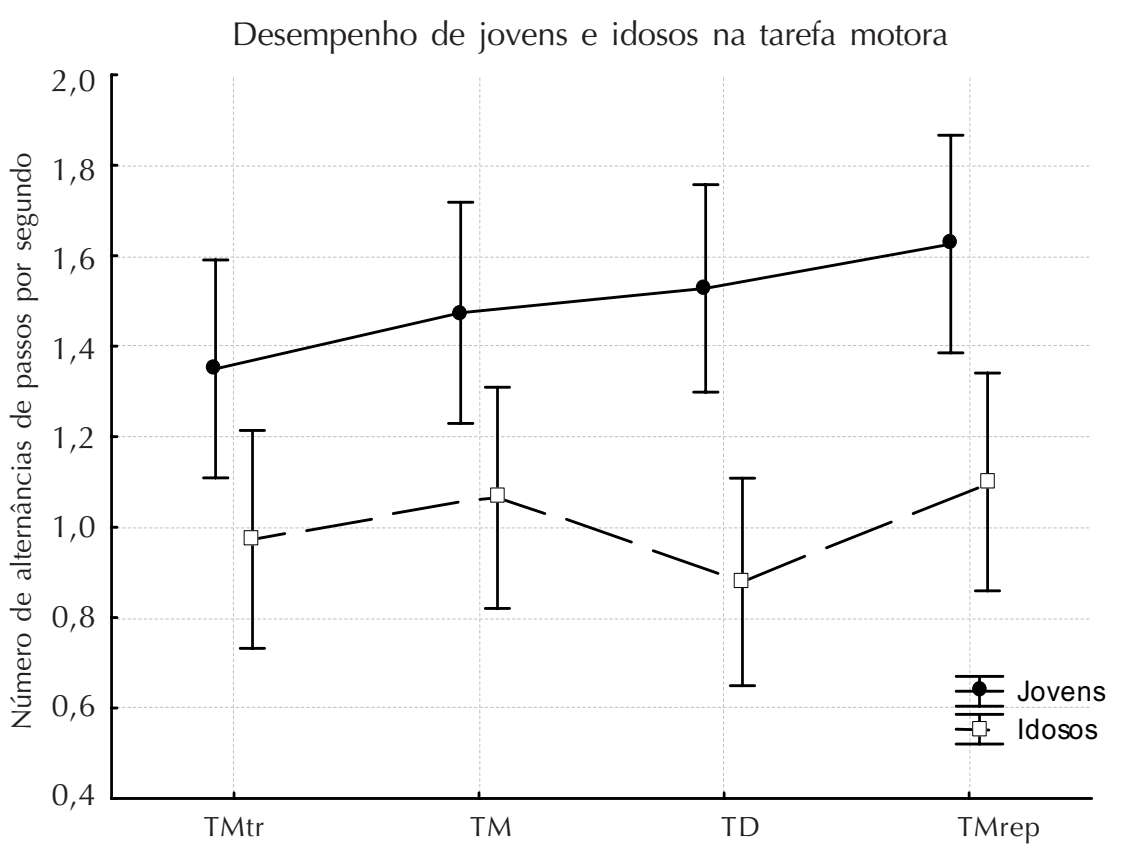

Figura 3 Desempenho médio de jovens e idosos na tarefa motora. TMtr = treino da tarefa motora; TM = tarefa motora; TD = tarefa motora associada à tarefa visual (tarefa-dupla); TMrep = repetição da tarefa motora para avaliar interferência do cansaço no desempenho

sos nessa situação. Logo, houve interferência negativa da tarefa visual sobre a tarefa motora para idosos, mas não para jovens.

\section{Correlações}

Foi criado um índice para verificar a interferência da tarefa visual sobre a tarefa motora: número de alternâncias de passos dados na TM menos o número de alternâncias na TD, tarefa motora associada à visual (índice 1). Esse índice foi correlacionado com o tempo-delta do TMT (tempo gasto na parte $\mathrm{B}$ menos tempo gasto na parte $A$ ), aqui chamado índice 2 , pelo teste de correlação de Spearman. Foi observada correlação significativa entre os índices $(r=0,451, p<0,05)$, ou seja: quanto menos passos o indivíduo tiver alternado durante a tarefa-dupla do que na tarefa motora isolada, menor o tempo a mais necessário para alternar números e letras no TMT-parte B do que o tempo requerido para seguir apenas a seqüência numérica no TMT-parte A.

O número de alternâncias de cada indivíduo no treino da tarefa motora (que foi o primeiro contato do sujeito portanto não houve interferência negativa da tarefa motora sobre a tarefa visual. Mas, embora a tarefa visual não tenha prejudicado o desempenho dos jovens na tarefa motora, esse prejuízo ocorreu para os idosos.

Assim como andar demanda alto custo de atenção em idosos ${ }^{13,14}$, a alternância de passos também parece ter essa característica. Como os idosos apresentam redução da habilidade de manter a postura estável, devido ao declínio da capacidade sensorial ${ }^{12}$, cognitiva e motora ${ }^{5,8,13,18}$, precisam alocar mais atenção ao controle postural, prejudicando assim a tarefa motora, sobretudo quando esta é associada à visual.

É importante mencionar o fato de, com a idade, ocorrer um aumento na dependência visual para atividades do dia-a-dia, como a marcha12. Foi solicitada a manutenção do olhar na tela do monitor durante todo o experimento e os idosos podem ter sido prejudicados por não terem podido usar a estratégia visual compensatória durante as tarefas motoras, tanto na isolada quanto na associada à visual.

Idosos apresentam prejuízo das funções executivas ${ }^{10,17}$, o que explica o fato de terem cometido mais erros na tarefa visual. Por outro lado, já que os idosos foram mais lentos na execução da tarefa motora na condição tarefadupla, tiveram mais tempo entre cada alternância para realizar a tarefa visual. Por isso, mesmo tendo mais dificuldade em dividir a atenção, conseDISCUSSÃO guiram manter o mesmo número de erros na tarefa visual nas condições tarefa-simples e tarefa-dupla.

Foi importante não ter sido atribuída relevância diferente a cada tarefa. Se uma tarefa tivesse sido estabelecida como primária e outra como secundária, não teria sido possível estudar a interferência da tarefa motora sobre a visual e vice-versa. O primeiro objetivo do presente estudo foi verificar eventual interferência negativa mútua entre as tarefas visual e motora. O segundo objetivo foi verificar diferenças no desempenho de jovens e idosos. Quanto aos dois objetivos, verificamos que a tarefa motora não prejudicou o desempenho da tarefa visual dos jovens nem dos idosos,
Tanto jovens quanto idosos apresentaram melhora na última tarefa motora, que visava verificar se o cansaço havia interferido no experimento: caso nessa tarefa (TMrep) apresentassem desempenho pior que nas anteriores, o cansaço do voluntário poderia ter interferido no resultado. Com a meIhora no desempenho, constata-se que houve aprendizado durante a realização da tarefa motora e que o cansaço não interferiu no resultado.

Nas execuções da tarefa motora simples (TMtr e TM), podem ter sido 
ativadas tanto as estruturas corticais como as subcorticais ${ }^{11}$. Ao ser associada à tarefa visual (tarefa-dupla), é possível que a tarefa motora tenha solicitado menos das estruturas corticais, conforme sugerido por Dubost et al. ${ }^{11}$, tendo utilizados circuitos subcorticais para deixar livre maior número de redes neuronais corticais para a tarefa visual. Assim, criaram-se novas estratégias e novos esquemas neurais para lidar com a situação de tarefa-dupla e novas áreas corticais podem ter sido ativadas para gerenciar simultaneamente as tarefas visual e motora ${ }^{28}$ Portanto, ao realizar novamente a tarefa-simples motora, o indivíduo teria contado com maior número de estratégias neurais. Isso pode complementar a explicação de melhor desempenho de jovens e idosos na última repetição da tarefa motora simples, após executá-la na condição tarefa-dupla. Embora a diferença de desempenho na tarefa motora antes e depois da tarefa-dupla não tenha alcançado nível significativo, houve maior velocidade na última realização da tarefa motora.
Este estudo teve como terceiro objetivo verificar se os testes realizados tinham correlação com testes validados que avaliam o mesmo comportamento, como o TMT e o TLCC. Quanto ao TMT, foi observada correlação com a tarefa-dupla: quanto menor o impacto da perturbação no TMT (ligar letras e números na parte $B$, ao invés de só números na parte $A$ ), menor o impacto da perturbação da tarefa visual sobre a motora (executar apenas a alternância de passos ou executá-la simultaneamente à discriminação de estímulos visuais). Essa correlação pode ser explicada pelo fato de os dois testes exigirem divisão de atenção, memória operacional e flexibilidade cognitiva.

A alternância de passos correlacionou-se ao TLCC pelo fato de ambos exigirem fatores em comum, como velocidade, habilidade de recrutar sinergias musculares alternadas e ajustes antecipatórios. Quanto mais rápido o indivíduo conseguiu completar o TLCC, maior foi sua velocidade de alternância de passos.
Por terem sido avaliadas nesse estudo apenas três vezes a tarefa visual, contra quatro vezes a tarefa motora, pode-se supor que, se tivesse havido uma quarta etapa visual, talvez fosse possível ter observado melhora no desempenho visual após a realização da tarefa-dupla, assim como foi observado na tarefa motora. Estudos futuros terão como objetivo investigar essa questão.

\section{CONCLUSÃO}

A tarefa motora não interferiu no desempenho da tarefa visual em jovens nem em idosos, mas a tarefa visual diminuiu a velocidade da tarefa motora entre os idosos; portanto, houve diferença entre o desempenho de jovens e idosos. Verificou-se ainda correlação entre os desempenhos nos testes de seqüência alfanumérica e levantar e caminhar cronometrado (TMT e TLCC) e nas tarefas propostas neste experimento.

\section{REFERENCIAS}

1 Cavanagh P, Alvarez GA. Tracking multifocal targets with multifocal attention. Trends Cogn Sci. 2005;9(7):349-54.

2 Eversheim $U$, Bock O. Evidence for processing stages in skill acquisition: a dual-task study. Learn Mem. 2001;8:183-9.

3 Herath P, Torkel K, Jeremy Y, Kathrin A, Per R. Neural correlates of dual-task interference can be dissociated from those of divided attention: an fMRI study. Cereb Cortex. 2001;11:796-805.

4 Yardley L, Gardner M, Bronstein A, Davies R, Buckwell D, Luxon L. Interference between postural control and mental task performance in patients with vestibular disorder and healthy controls. J Neurol Neurosurg Psychiatry. 2001;71(1):48-52.

5 Brauer SG, Woollacott M, Shumway-Cook A. The interacting effects of cognitive demand and recovery of postural stability in balance-impaired elderly persons. J Gerontol A Biol Sci Med Sci. 2001;56(8):M489-96.

6 Steinman SB, Steinmann BA. Vision and attention I: current models of visual attention. Optom Vis Sci. 1998;75(2):146-55.
7 Roland PE, Zilles K. Structural divisions and functional fields in human cerebral cortex. Brain Res Brain Res Rev. 1998;26:87-105.

8 Ble A, Volpato S, Zuliani G, Guralvinik JM, Bandinelli S, Lauretani $F$ et al. Executive function correlates with walking speed in older persons: the InCHIANTI study. J Am Geriatr Soc. 2006;53:410-5.

9 Broman AT, West S, Munoz B, Bandeen-Roche K, Rubin G, Turano KA. Divided visual attention as a predictor of bumping while walking: the Salisbury Eye Evaluation. Invest Ophtalmol Vis Sci. 2004;45(9):2955-60.

10 Coppin AK, Shumway-Cook A, Saczynski J, Patel KV, Ble A, Ferrucci L, et al. Association of executive function and performance of dual-task physical tests among older adults: analyses from the InChianti study. Age Ageing. 2006;35(6):619-24.

11 Dubost V, Kressig RW, Gonthier R, Herrmann FR, Aminian K, Najafi B, et al. Relationship between dual-task related changes in stride velocity and stride time variability in healthy older adults. Hum Mov Sci. 2006;25:372-82. 


\section{Referências (cont.)}

12 Jamet M, Deviterne D, Gauchard GC, Vançon G, Perrin PP. Age-related part taken by attentional cognitive process in standing postural control in a dual-task context. Gait Posture. 2006;25(2):179-84.

13 Silsupadol P, Siu KC, Shumway-Cook A, Woollacott $\mathrm{H}$. Training of balance under single- and dual-task conditions in older adults with balance impairment. Phys Ther. 2006;86:269-78.

14 Sparrow WA, Bradshaw EJ, Lamoureux E, Tirosh O. Aging effects on attention demands of walking. Hum Mov Sci. 2002;21:961-72.

15 Holtzer R, Friedman R, Lipton RB, Katz M, Xue X, Verghese J. The relationship between specific cognitive functions and falls in aging. Neuropsychology. 2007;21(5):540-8.

16 Ball K, Owsley C. J. The useful field of view test: a new technique for evaluating age related declines in visual function. Am Optom Assoc. 1993;64(1):71-9.

17 Oxley JA, Ihsen E, Fildes BN, Charlton JL, Day RH. Crossing roads safely: an experimental study of age differences in gap selection by pedestrians. Accid Anal Prev. 2005;37(5):962-71.

18 Alexander NB, Ashton-Miller JA, Giordani B, Guire $K$, Schultz AB. Age differences in timed accurate stepping with cognitive and visual demand: a walking trail making test. J Gerontol A Biol Sci Med Sci. 2005;60(12):1558-62.

19 Rebok GW, Carlson MC, Langbaum JC. Training and maintaining memory abilities in healthy older adults: traditional and novel approaches. J Gerontol B Psychol Sci Soc Sci. 2007;62(1):53-61.
20 Lezak MD. Neuropsychological assessment. New York: Oxford University Press; 1995.

21 Nevitt MC, Cummings SR, Hudes ES. Risk factors for injurious falls: a prospective study. J Gerontol. 1991;46:M164-70.

22 Podsiadlo D, Richardson S. The timed up \& go: a test of basic functional mobility for frail elderly persons. J Am Geriatr Soc. 1991;39:142-8.

23 DiFabio RP, Kurzevski WM, Jorgenson EE et al. Footlift asymmetry during obstacle avoidance in high-risk elderly. J Am Geriatr Soc. 2004;52:2088-93.

24 Turano KA, Broman AT, Bandeen-Roche K, Munoz B, Rubin GS, West S. Association of visual field loss and mobility performance in older adults: Salisbury eye evaluation study. Optom Vis Sci. 2004;81(5):298-307.

25 Silveira RR, Partezani RA, Costa Jr ML. Idosos que foram vítimas de acidentes de trânsito no município de Ribeirão Preto, SP, em 1998. Rev Latino-Am Enfermagem. 2002;10(6):213-20.

26 Brucki SMD, Nitrini R, Caramelli P, Bertolucci PHF, Okamoto IH. Sugestões para o uso do miniexame do estado mental no Brasil. Arq Neuropsiquiatr. 2003;61(3B):777-81.

27 Littlewood R, Johnson G, House P. Vision testing in atrophic macular degeneration. Aust NZ J Ophtalmol. 1996;24(1):47-51.

28 Erickson KI, Colcombe SJ, Wadhwa R, Bherer L, Peterson MS, Scalf PE, et al. Training-induced functional activation changes in dual-task processing: an FMRI study. Cereb Cortex. 2007;17(1):192-204. 